\title{
Elucidating the molecular characteristics of organogenesis in human embryos
}

\author{
Xin Geng and Guillermo Oliver*
}

\begin{abstract}
A transcriptomic analysis of early human

organogenesis reveals the molecular signature of

these processes and provides a valuable resource

for identifying and comparing crucial regulators of

mammalian embryogenesis.
\end{abstract}

Embryogenesis is a process by which embryonic cells responding to extrinsic signals lose their totipotency their ability to develop into any cell type - and gradually restrict their development potential to a specific lineage. Mammalian embryogenesis starts when the totipotent zygote divides and its descendants progressively restrict their development potential to become either extraembryonic tissue or inner cell mass. Inner mass cells are the pluripotent progenitors from which the entire embryo, other than some extraembryonic tissues, will be derived. During gastrulation, pluripotent progenitors further confine their fates to one of the three primary germ layers (ectoderm, mesoderm or endoderm). After gastrulation, organogenesis starts with the formation of organ primordia and the subsequent differentiation of various cell types within those organs.

Anatomical aspects of mammalian embryogenesis have been the subject of detailed morphologic characterization over the centuries. The molecular profile underlying this process has been a focus of research more recently, but is still poorly understood. For example, the genes that maintain the pluripotency of progenitor cells and that regulate the stepwise differentiation of progenitors into various cell types are only now starting to be identified. Because this research is mostly done in model organisms such as the mouse, it is important to verify that such results extend to humans, and so could eventually be

*Correspondence: guillermo.oliver@stjude.org

Department of Genetics and Tumor Cell Biology, St Jude Children's Research Hospital, 262 Danny Thomas Place, Memphis, TN 38105-3678, USA considered for clinical application. Because of obvious ethical concerns, human embryonic tissues are hard to obtain; therefore, our ability to extrapolate knowledge from mice to humans is limited.

\section{The human embryo transcriptome}

With some of the above problems in mind, the genomewide transcriptomic profiling of early post-implantation human embryos, published recently in Developmental Cell by Fang et al. [1], will be particularly valuable. Human embryos from six consecutive Carnegie stages (S9 to S14), which cover the first third of the period of organogenesis, were used for this analysis. Carnegie stages for human embryos are defined by external and internal anatomical developmental criteria and run from stage 1 (zygote) to stage 23 (around 56 days gestation). During stages 9 to 14 and following the completion of gastrulation, the neural plate folds to form the neural tube and brain, and structures and organs such as somites, heart and limb buds start to develop. Embryos at these stages were pooled for Affymetrix expression profiling to minimize variation and were run in triplicate for consistency. Not surprisingly, the authors found that, as with the transcriptome of early mouse embryos [2], the most dramatic change in gene-expression profile occurred as the human embryos completed gastrulation and initiated organogenesis [1] (around embryonic day 8 (E8.0) in mouse and at the S9-S10 transition in human embryos). This drastic change at the transcriptome level from S9 to S10 is most likely to be because numerous organ primordia start to develop between S10 and S12 [1]. Using available data-analysis resources, including gene clustering and enrichment analysis, the authors identified six clusters (clusters 1 to 6) of genes displaying similar expression patterns. Clusters 1,2 and 3 were similar in that the expression of their genes appeared to be gradually repressed as development proceeded, indicating a gradual decrease in 'stemness'. Concomitant with the increasing diversity of cell types, the expression of genes in clusters 5 and 6 (which included numerous transcription factor genes) gradually increased; these clusters include a significant number of genes that have been identified as organogenesis-specific in mice [2]. 
Using a phenotype-gene ontology analysis, Fang et al. also determined that defects in genes in clusters 1 through 3 were, in general, associated with embryonic lethality and defective embryogenesis, but rarely with genetic disorders, whereas defects in genes in clusters 5 and 6 were mainly related to postnatal lethality, various organ defects and multiple genetic disorders.

A valuable aspect of this work is that it will enable direct comparisons of available mammalian transcriptomes. This type of comparative analysis is highly relevant, considering that mice are one of the main experimental models but humans are the targets of potential diagnostic and therapeutic approaches. Although humans and mice share $85 \%$ of their genes and undergo a similar process of embryogenesis, differences in gene regulation are most likely the leading cause of obvious differences between species [3]. Zhang et al. [4] recently highlighted some important species-specific differences in the role of the transcription factor Pax6 in the specification of the neurectoderm - the ectodermal cells that will develop into the animal's nervous system. They determined that although Pax6 activity is not required for neuroectoderm specification in mice, its expression follows that of the transcription factor Sox1, an early marker for neuroectoderm. Interestingly, in humans the situation is the converse - that is, a specific isoform of PAX6 is expressed before SOX1 and is required for neuroectoderm specification. Zhang et al. argue that the early expression of $P A X 6$ in humans (similar to their previous results with rhesus monkey embryonic stem cells), may have been a step in the evolution of the highly evolved forebrain of primates [4]. In their study, Fang et al. [1] identified a set of genes that are expressed early in human embryonic development but have not yet been implicated in early mouse development. In future studies, it will be important to determine whether these findings implicate important species-specific differences in the regulation of organ development.

\section{Charting the loss of pluripotency}

Fang et al. also took advantage of the dataset they generated to compare the gene-expression profile of human embryos with that of human embryonic stem cells (hESCs) maintained in culture. They found that approximately $20 \%$ of the genes in the clusters whose expression was decreasing at S9 to S11 (when organogenesis is beginning and pluripotency is being lost) are also expressed in hESCs, and that many of these genes are likely to be regulated by pluripotency-promoting transcription factors such as POU5F1 (OCT4), SOX2, and NANOG. In these clusters, pluripotency-promoting genes are coexpressed with differentiation-promoting genes that are most likely involved in the initiation of organogenesis. A similar expression pattern was observed during the transcriptome analysis of early mouse embryogenesis, in which pluripotency genes were found to be coexpressed with the regulators of gastrulation. These results validate the gene-expression data collected from studies of hESC differentiation in vitro. At the early stages of hESC differentiation, pluripotency-promoting and lineage-specific genes are coexpressed (lineage priming). As the expression of pluripotency genes wanes, the cells gradually lose their ability to self-renew and differentiate into specific cell types [5]. Consistent with this notion, as development proceeds, genes in clusters 5 and 6 (expressed during S12 to S14) - which are most probably involved in the differentiation of various cell types and the formation of organs - become upregulated. During S12 to S14, structures such as the nervous system, the heart and the somites develop further; meanwhile, the primordium of other structures, such as lungs and ureteric buds, start to emerge. These genes are underrepresented in hESCs and are likely to be regulated by organogenesis-related transcription factors such as the heart-specific NKX2-5, the skeletal-specific SOX5, the nervous system-specific OCT1 and BRN2, and the muscle-specific MEF2.

Although transcriptome analysis will be invaluable to researchers trying to understand the early stages of mammalian organogenesis, it is not without limitations. Transcriptional status alone is not a sufficient indicator of a particular gene product's activity; protein expression can also be regulated at the translational level and by numerous posttranslational modifications and proteinprotein interactions. For example, NKX2-5 belongs to cluster 3 and its expression is dramatically upregulated from S9 to S10; however, its target genes are only enriched in clusters 5 and 6 (expressed during S12 to S14). Similarly, although the relative expression levels of SOX5 RNA do not change during early organogenesis, its target genes are enriched in clusters 5 and 6 . To overcome these limitations, at least partially, Fang et al. have constructed a molecular network assembling the interacting genes. Because this network analysis is mostly hypothetical, complementary approaches such as proteomics, and even more importantly, hypothesis-driven research, will be necessary to validate these results.

Human organogenesis starts at Carnegie stage 9 and ends at around stage 23. The study from Fang et al. covers the first third of human organogenesis from Carnegie stage 9 to stage 14. A complementary study using a strategy similar to that of Fang et al. has analyzed the transcriptome of human embryos from Carnegie stage 10 to stage 23, which, combined with the study from Fang et al., covers the entire period of organogenesis in human embryos [6]. Yet other studies have analyzed the transcriptomes of human oocytes, hESCs and human preimplantation embryos (blastocysts) [7-9]. A few missing 
pieces, such as the gene-expression profile of the human gastrula, will help complete the molecular characterization of early human embryogenesis. These valuable resources will help elucidate the basic principles of embryogenesis, expand our understanding of speciesspecific differences during development, and eventually help engineer hESCs in culture for therapeutic purposes.

Published: 27 August 2010

\section{References}

1. Fang $H$, Yang Y, Li C, Fu S, Yang Z, Jin G, Wang K, Zhang J, Jin Y: Transcriptome analysis of early organogenesis in human embryos. Dev Cell 2010, 19:174-184.

2. Mitiku N, Baker JC: Genomic analysis of gastrulation and organogenesis in the mouse. Dev Cell 2007, 13:897-907.

3. Waterston RH, Lindblad-Toh K, Birney E, Rogers J, Abril JF, Agarwal P, Agarwala R, Ainscough R, Alexandersson M, An P, Antonarakis SE, Attwood J, Baertsch R, Bailey J, Barlow K, Beck S, Berry E, Birren B, Bloom T, Bork P, Botcherby M, Bray N, Brent MR, Brown DG, Brown SD, Bult C, Burton J, Butler J, Campbell RD, Carninci $P$, et al. Initial sequencing and comparative analysis of the mouse genome. Nature 2002, 420:520-562.

4. Zhang X, Huang CT, Chen J, Pankratz MT, Xi J, Li J, Yang Y, Lavaute TM, Li XJ,
Ayala M, Bondarenko Gl, Du ZW, Jin Y, Golos TG, Zhang SC: Pax6 is a human neuroectoderm cell fate determinant. Cell Stem Cell 2010, 7:90-100.

5. Laslett AL, Grimmond S, Gardiner B, Stamp L, Lin A, Hawes SM, Wormald S, Nikolic-Paterson D, Haylock D, Pera MF: Transcriptional analysis of early lineage commitment in human embryonic stem cells. BMC Dev Biol 2007 7:12.

6. Yi H, Xue L, Guo MX, Ma J, Zeng Y, Wang W, Cai JY, Hu HM, Shu HB, Shi YB, Li WX: Gene expression atlas for human embryogenesis. FASEB J 2010, doi:10.1096/fi.10-158782.

7. Kocabas AM, Crosby J, Ross PJ, Otu HH, Beyhan Z, Can H, Tam WL, Rosa GJ, Halgren RG, Lim B, Fernandez E, Cibelli JB: The transcriptome of human oocytes. Proc Natl Acad Sci USA 2006, 103:14027-14032

8. Assou S, Le Carrour T, Tondeur S, Ström S, Gabelle A, Marty S, Nadal L, Pantesco V, Réme T, Hugnot JP, Gasca S, Hovatta O, Hamamah S, Klein B, De Vos J: A meta-analysis of human embryonic stem cells transcriptome integrated into a web-based expression atlas. Stem Cells 2007, 25:961-973.

9. Dobson AT, Raja R, Abeyta MJ, Taylor T, Shen S, Haqq C, Pera RA: The unique transcriptome through day 3 of human preimplantation development. Hum Mol Genet 2004, 13:1461-1470.

\section{doi:10.1186/gb-2010-11-8-130}

Cite this article as: Geng X, Oliver G: Elucidating the molecular

characteristics of organogenesis in human embryos. Genome Biology 2010,

11:130. 